University of Nebraska - Lincoln

DigitalCommons@University of Nebraska - Lincoln

6-1-2001

\title{
Molecular dynamics simulation of supersaturated vapor nucleation in slit pore. II. Thermostatted atomic-wall model
}

\author{
K.T. Kholmurodov \\ Institute of Physical and Chemical Research (RIKEN), Hirosawa 2-1, Wako, Saitama 351-0198, Japan \\ K. Yasuoka \\ Keio University \\ Xiao Cheng Zeng \\ University of Nebraska-Lincoln, xzeng1@unl.edu
}

Follow this and additional works at: https://digitalcommons.unl.edu/chemzeng

Part of the Chemistry Commons

Kholmurodov, K.T.; Yasuoka, K.; and Zeng, Xiao Cheng, "Molecular dynamics simulation of supersaturated vapor nucleation in slit pore. II. Thermostatted atomic-wall model" (2001). Xiao Cheng Zeng Publications. 44.

https://digitalcommons.unl.edu/chemzeng/44

This Article is brought to you for free and open access by the Published Research - Department of Chemistry at DigitalCommons@University of Nebraska - Lincoln. It has been accepted for inclusion in Xiao Cheng Zeng Publications by an authorized administrator of DigitalCommons@University of Nebraska - Lincoln. 


\title{
Molecular dynamics simulation of supersaturated vapor nucleation in slit pore. II. Thermostatted atomic-wall model
}

\author{
K. T. Kholmurodova) \\ Computational Science Division, Advanced Computing Center, The Institute of Physical and Chemical \\ Research (RIKEN), Hirosawa 2-1, Wako, Saitama 351-0198, Japan \\ K. Yasuoka \\ Department of Mechanical Engineering, Keio University, Yokohama 223-8522, Japan \\ X. C. Zeng ${ }^{\text {b) }}$ \\ Department of Chemistry, University of Nebraska-Lincoln, Lincoln, Nebraska 68588
}

(Received 30 January 2001; accepted 15 March 2001)

\begin{abstract}
Molecular dynamics simulations of nucleation of a supersaturated Lennard-Jones vapor in slit nanopores are carried out. In this study we extend a previous work [K. Yasuoka, G. T. Gao, and X. C. Zeng, J. Chem. Phys. 112, 4279 (2000)] in that the walls of the slit are treated as actual atomic walls serving as both the confining solid surfaces and a thermostat. The walls are fixed in place in a fcc lattice structure and wall atoms are subjected to a stiff biharmonic potential thereby bounded to lattice sites. The two walls of the slit have an identical surface [fcc (100)], but different strength of attractive interaction with the vapor particles-one is strongly adsorbing and another is weakly adsorbing. Heterogeneous nucleation of the supersaturated vapor in the slit is investigated and events of nucleus formation are monitored in real time. A comparison with the previous simulation (using rigid structureless walls) leads to useful insight into the influence of the wall model to the nucleus formation. In particular, it is found that although the adsorbed particles on the structureless wall diffuse faster than those on the atomic wall, the rate of nucleus formation on the structureless wall is actually about one order of magnitude lower. A detailed analysis of particle and cluster-formation flux indicates that the rate of nucleus formation on the wall is more sensitive to the kinetics of adsorption of gas particles onto the wall than the diffusion rate of adsorbed particles. The higher flux of cluster formation on the atomic wall is apparently due to the higher rate of deposition of monomers onto the wall. (C) 2001 American Institute of Physics. [DOI: 10.1063/1.1370057]
\end{abstract}

\section{INTRODUCTION}

Confined geometries affect the phase behavior of fluids. In a slit nanopore, for example, an undersaturated vapor can condense into a liquid if the liquid partially wets two walls of the slit (i.e., with a contact angle of less than $90^{\circ}$ ). This is known as capillary condensation. ${ }^{1-5}$ Even if the walls are nonwetting, the nanoscale confinement can still lead to a shift of the bulk binodal curve,${ }^{6,7}$ thereby affecting the condition of condensation. Our principal aim in this work is to investigate heterogeneous nucleation of a supersaturated vapor in between two planar solid walls: one strongly adsorbing and one weakly adsorbing. A better understanding of the nucleation at the molecular level is of great importance to many engineering applications, ${ }^{8}$ e.g., separation processes and gas field technology, among others. It is known that the transition from a metastable supersaturated vapor to a thermodynamically stable liquid must proceed through a dropletformation process, namely, nucleation. Nucleation is a thermally activated process and the rate of nucleus formation depends exponentially on barrier height to nucleation. The barrier is characterized by the free energy of formation of the

\footnotetext{
a)Permanent address: Laboratory of Information Technologies, Joint Institute for Nuclear Research, Dubna, Moscow Region, 141980, Russia

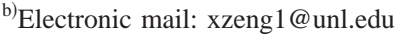

critical nucleus. ${ }^{9}$ Thus, nucleation dynamics can be sensitive to a small change in the environmental conditions.

In our previous molecular dynamics simulation of vapor nucleation in slit pores ${ }^{7}$ (hereafter referred to as paper I), the location of the nucleus formation as well as the kinetic path to the nucleus formation have been investigated as we varied the strength of attraction between a rigid structureless wall and vapor particles. It was found that this change in attractive interaction can strongly affect the process of nucleus formation: if the attraction is weak (i.e., a weakly adsorbing wall), nuclei tend to form in the interior of the pore, whereas if the attraction is strong (i.e., a strongly adsorbing wall) the nucleus formation originates from two sources: surface diffusion of adsorbed particles on the wall and the deposition of clusters formed in the interior of the pore. An appreciable enhancement of the rate of nucleus formation was found in all nanopores studied. The observation of surface diffusion of adsorbed particles as one of the important mechanisms in the nucleus formation is consistent with the experimental finding by Maa and co-workers. ${ }^{10-12}$.

In our previous simulation (paper I) the condensation heat near the walls are removed simply by scaling velocity of the target particles because the rigid structureless walls cannot directly conduct the heat. In this work we extend the previous study by using a more realistic wall model. We 
replace the rigid structureless walls by thermostatted atomic walls. The walls are fixed in place in a fcc lattice structure by a combination of the restoring tethering forces and constraint mechanism. The restoring potential confines wall atoms to their lattice sites at the equilibrium state. Since the walls are thermostatted, the latent heat generated during nucleus formation can be dissipated through the walls, like in real-world experiments. ${ }^{10-14}$ Moreover, the atomic-wall model allows a more realistic simulation of the surface diffusion process of adsorbed particles toward nucleus formation. The two surfaces of the pore are identical in structure, but have different strengths of the attractive interaction with the vapor particles: one is a strongly adsorbing wall and the other is a weakly adsorbing wall. The rate of nucleus formation is evaluated by monitoring the molecular dynamics configurations of the system in real time and by identifying the shortlived nucleus-formation events. To gain more insight on the wall-model effects to the nucleus formation the results of the present study (using the atomic-wall model) are compared with those in paper I (using the smooth-wall model). For this purpose, all calculations for the smooth-wall system were reexamined using the exactly same simulation conditions as for the atomic-wall system.

\section{SYSTEM AND SIMULATION METHOD}

\section{A. Vapor}

The binary vapor mixture consists of 4320 target and 4320 carrier-gas particles. As in paper I, the carrier gas is used to avoid unnatural energy exchanges in the process of nucleus formation and to release the latent heat generated during the nucleus formation. To this end, the carrier gas is connected to a hypothetical heat bath by using a NoséHoover (or Berendsen) thermostat to regulate the temperature of the system. ${ }^{7}$

The target-target interaction potential $U_{\mathrm{tt}}(r)$ is a Lennard-Jones (LJ) type, i.e.,

$$
U_{\mathrm{tt}}(r)=4 \epsilon\left[\left(\frac{\sigma}{r}\right)^{12}-\left(\frac{\sigma}{r}\right)^{6}\right],
$$

where $r$ is the interparticle distance. For argon, the LJ potential parameters are $\epsilon=0.0103 \mathrm{eV}$ and $\sigma=3.405 \AA$. The particle mass $m=6.63 \times 10^{-26} \mathrm{~kg}$. The potential between target-carrier and carrier-carrier particles is a soft-core type (no interparticle attraction),

$$
U_{\mathrm{tc}}(r)=U_{\mathrm{c}}(r)=4 \epsilon\left(\frac{\sigma}{r}\right)^{12} .
$$

The soft-core potential parameters $\epsilon$ and $\sigma$, and the carriergas particle mass $m$ are set to be the same as those of target particles. Hereafter we use $\sigma, \epsilon$, and $m$ as the units of length, energy, and mass, respectively. The unit of time is then $\tau$ $=\sqrt{m \sigma^{2} / \epsilon}=2.15 \mathrm{ps}$. In the molecular dynamics (MD) simulation, the time step is $\tau_{s}=0.0023255 \tau=5$ fs and the cutoff distance for particle interaction is $4.5 \sigma$. The interior volume of the pore is $72 \sigma \times 72 \sigma \times 36 \sigma$, and thus the number density of target particles at the initial state is $\rho_{v}=2.315$ $\times 10^{-2} \sigma^{-3}$, the same as used in the study of homogeneous nucleation of a bulk vapor. ${ }^{15}$
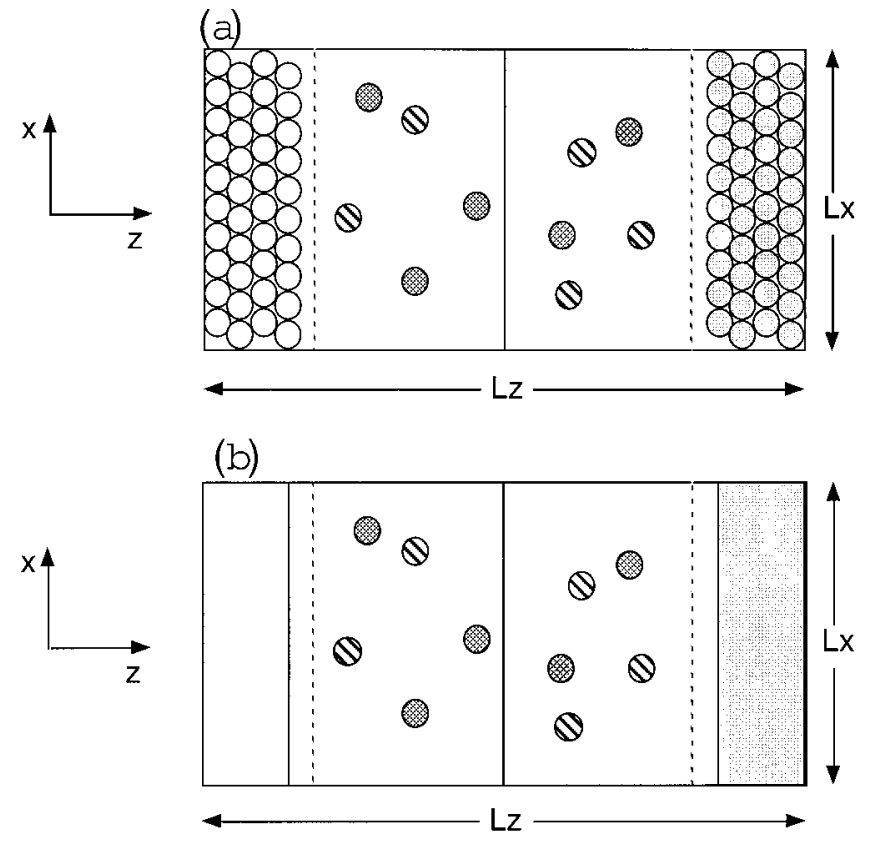

(c)

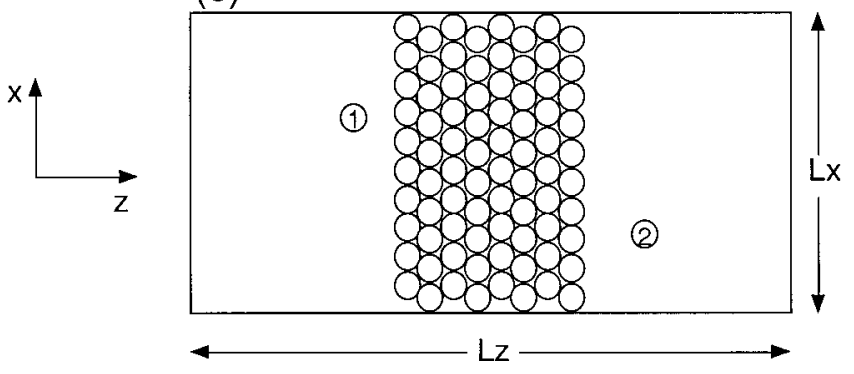

FIG. 1. A schematic plot of the simulated (a) atomic-wall and (b) smoothwall system. The two types of circles in the middle region denote the target and carrier-gas particles. The open (left) and dark (right) circles for the atomic-wall model and the white (left) and dark (right) areas for the smoothwall model represent the weakly and strongly adsorbing walls. The $y$ axis is normal to the page. (c) A schematic plot of the real simulation cell employed in the DL_POLY code. During the simulation, the wall itself can play a dual role, i.e., both wall 1 and wall 2 . If a vapor particle (circled 1) is within a cutoff distance 4.5 from the left surface of the central wall, the particle will experience a potential field of wall 1 . If a vapor particle (circled 2 ) is within a distance 4.5 from the right surface of the wall, the particle will experience a potential field of wall 2. Because of the potential cutoff distance 4.5 , effectively, vapor particles can only interact with four layers of wall at most. Thus, the simulation cell is computationally almost equivalent to that displayed in (a) but free of the contact-heat-resistance problem because the wall is homogeneous.

\section{B. The solid walls}

A wall of the slit consists of eight layers of a facecentered cubic (fcc) (100) lattice of 25600 atoms (3200 atoms per layer). The number density of wall atoms, given the surface area $72 \sigma \times 72 \sigma$, is then $\rho_{w}=0.69 \sigma^{-3}$. In order to efficiently simulate the heterogeneous nucleation, we let one wall of the slit be strongly adsorbing (wall 1) while the opposing wall is weakly adsorbing (wall 2). For this purpose, atoms of wall 1 have an energy parameter $\epsilon_{w_{1}}=\epsilon$ while atoms of wall 2 have $\epsilon_{w_{2}}=0.1 \epsilon$. A schematic plot of the simulated atomic- and smooth-wall systems are shown in Figs. 1 (a) and $1(\mathrm{~b})$, respectively.

The energy and size parameters of wall-vapor potential 
are given by the Lorentz-Berthelot combining rules, i.e., $\epsilon_{w, \text { gas }}=\sqrt{\epsilon_{w} \epsilon_{\mathrm{gas}}}$, and $\sigma_{w, \mathrm{gas}}=\left(\sigma_{w}+\sigma_{\mathrm{gas}}\right) / 2$. Thus, $\epsilon_{w_{1}}$,gas $=\epsilon($ wall 1$), \epsilon_{w_{2}, \text { gas }}=(0.1)^{1 / 2} \epsilon$ (wall 2), and $\sigma_{w}=\sigma_{\text {gas }}=\sigma$, where the subscript gas stands for both target and carrier-gas particles. The energy and size parameters of wall-carrier gas potential which is a soft-core type can be given in a similar fashion.

To simulate thermostatted atomic walls we adopted a method developed previously by several groups. ${ }^{16-19}$ Specifically, the walls are fixed in place of a fcc lattice structure by a combination of a tethering force and a constraint mechanism. To restrict wall atoms to be near their nominated lattice sites, the wall atoms, besides of LJ potential, are also subjected to a tethering, biharmonic potential of the form

$$
U_{\text {teth }}(r)=\frac{1}{2} k r^{2}+\frac{1}{4} k^{\prime} r^{4} \text {. }
$$

Under this restoring potential the wall atoms vibrate only around their lattice sites. In the present MD simulations, no near-wall target-particle velocity scaling is needed to model heat conduction through the walls. The atomistic vibration allows the heat conduction through the walls in a more natural way.

In Eq. (3), we chose the values $k=150$ and $k^{\prime}=200$ after examining a number of $k$ and $k^{\prime}$ values (including the values $k^{\prime}=0$, and $k=1.5 ; 15 ; 150$, etc). With the selected values of $k$ and $k^{\prime}$ we found the wall state is well behaved during the simulation. Before the vapor and wall are brought into contact, the wall is equilibrated first at a reduced temperature $T=0.67$ (the reduced temperature is in units of $\epsilon / k_{\mathrm{B}}$, where $k_{\mathrm{B}}$ is the Bolzmann constant) with using a slab boundary condition in $(x, y)$ directions.

\section{Molecular dynamics simulation}

The MD simulations of vapor nucleation were carried out on a Fujitsu VPP700 vector computer by using a DL_POLY_2.11 package. $^{20}$ Since this software package was not developed specifically for vector computers like VPP700, some optimizations of DL_POLY code for the vector computer have been undertaken. Significant performance enhancements have been achieved and the results have been reported elsewhere. ${ }^{21}$

In practice, one can straightforwardly employ a simulation cell, as shown in Fig. 1(a) into the DL_POLY code. However, this is not the most efficient way to maximize the usage of the given wall size (25600 atoms) because the wall size has to be split into two: one for wall 1 and one for wall 2. As such, only one-half of the wall size (i.e., wall 1 with 12800 atoms) would be efficiently used in the simulation of heterogeneous nucleation. Moreover, a problem of contact-heat resistance (between wall 1 and periodic images of wall 2, or vice versa) can be a concern. ${ }^{18,19}$ In reality, we implemented a simulation technique such that the simulation cell is computationally nearly the same as that displayed in Fig. 1(a). The simulation cell includes only one eight-layer wall at the center with the normal direction of the wall along the $z$ axis [see Fig. 1(c)]. Since the periodic boundary conditions are applied in all three spatial directions, the nearest
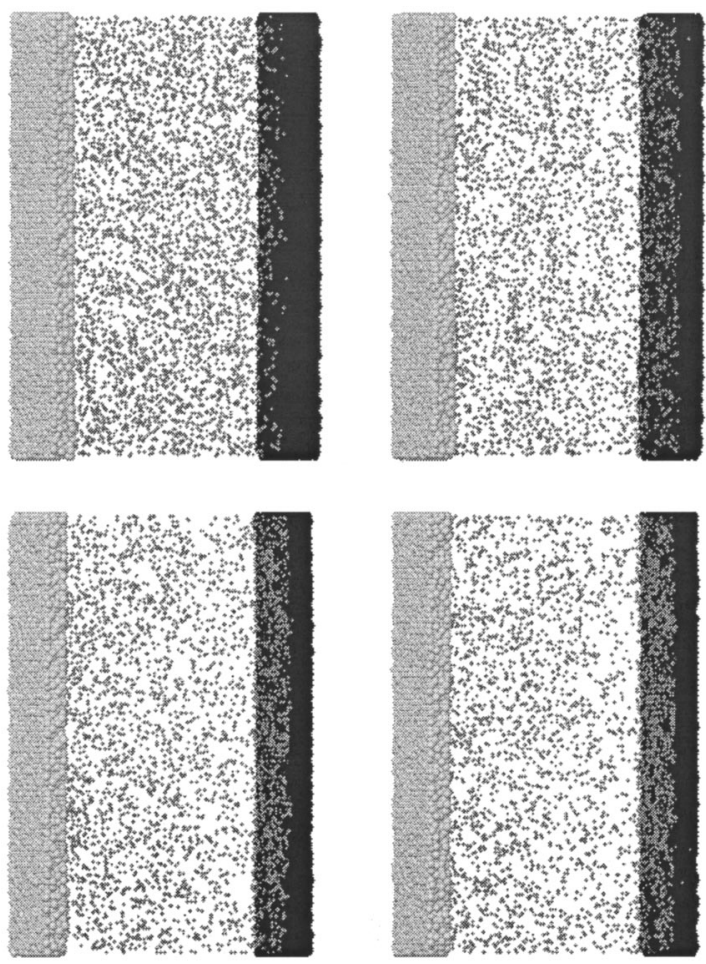

FIG. 2. Four sequential snapshots of the MD simulation. The carrier-gas particles are not shown. The slit is slightly rotated around the $x$ axis so that target-particle adsorption on the strongly adsorbing wall (dark) can be seen.

periodic images of the wall will play the role of opposing walls. In the course of the simulation, the wall itself can play a dual role, that is, both wall 1 and wall 2. If a vapor particle is within a cutoff distance 4.5 from the left surface of the central wall the particle will experience a potential field of wall 1. Similarly, a vapor particle will experience a potential field of wall 2 if the particle is within a distance 4.5 from the right surface of the wall. Because of the potential cutoff distance 4.5 , effectively, vapor particles can only interact with four layers of wall at most, regardless whether the wall acts as wall 1 or wall 2 . In this way, the simulation cell implemented in the DL_POLY code is computationally nearly identical to that displayed in Fig. 1(a), but free of the contactheat-resistance problem because the wall is a homogeneous single-component wall.

The simulation cell dimensions $\left(L_{x}, L_{y}, L_{z}\right)$ are assigned the following values: $L_{x}=L_{y}=72 \sigma, L_{z}=44.3 \sigma$, where $L_{z}$ includes the thickness of the wall. After separate equilibration, the vapor and the solid walls are brought together as a whole system. The vapor, first equilibrated at $T=1.50$, undergoes a temperature quench to a supersaturated state at $T$ $=0.67$ and then is brought into contact with the solid wall (equilibrated at $T=0.67$ ). The entire system then evolves at the given temperature for about $100 \tau$.

\section{SIMULATION RESULTS}

For the purposes of analysis we describe below the results for the "atomic-wall model", along with the reanalyzed results for the "smooth-wall model." For both wall-model systems the corresponding calculations were performed at the same simulation conditions. 

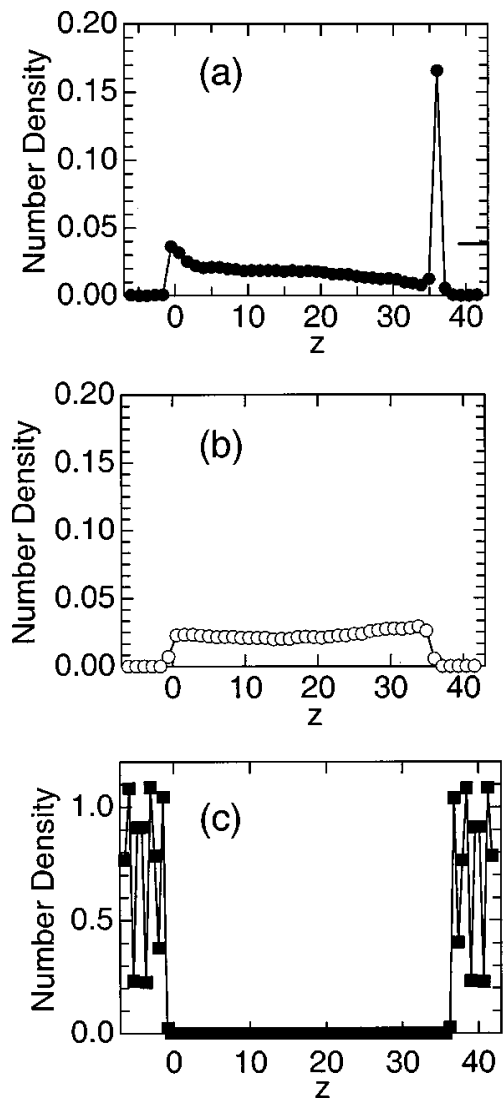

FIG. 3. The density profiles in the $z$ direction at a steady state for the (a) target, (b) carrier-gas, and (c) wall particles (the atomic-wall model).

\section{A. Snapshots}

In Figs. 2(a)-2(d), four sequential MD configurations of the system are displayed. The first snapshot corresponds to the intial state, right after the vapor and the solid walls are brought into contact. The next three snapshots in Figs. 2(b)2(d) show the evolution of the system at the moment of $t$ $=N_{s} \tau_{s}, 3 N_{s} \tau_{s}$, and $4 N_{s} \tau_{s}$, respectively, where $N_{s}=5000$. The carrier-gas particles are not shown in the figures. It can be seen from Fig. 2 that nucleation events take place mainly near the strongly adsorbing wall (wall 1). As a result, the local target-particle density near wall 1 is much higher than the overall mean density.

\section{B. The density and temperature profiles}

For the sake of numerical analysis we divided the inner space between the walls of the slit into several sections in the $z$ direction, each section having a width of about one $\sigma$. Numerical data, e.g., the particle positions and velocities in each section, are then collected. Moreover, in each section, the local density and temperature at each time step for both the vapor and wall are evaluated. In Figs. 3(a)-3(c) the density profiles (in the $z$ direction) of the target, carrier-gas, and wall particles at a steady state are presented, respectively (for the atomic-wall model). In Figs. 4(a)-4(c) the corresponding temperature profiles of the target, carrier-gas, and wall particles at the same steady state are shown. One can see that the density of the target particles near the strongly adsorbing wall is much higher (about five times larger) than the aver-
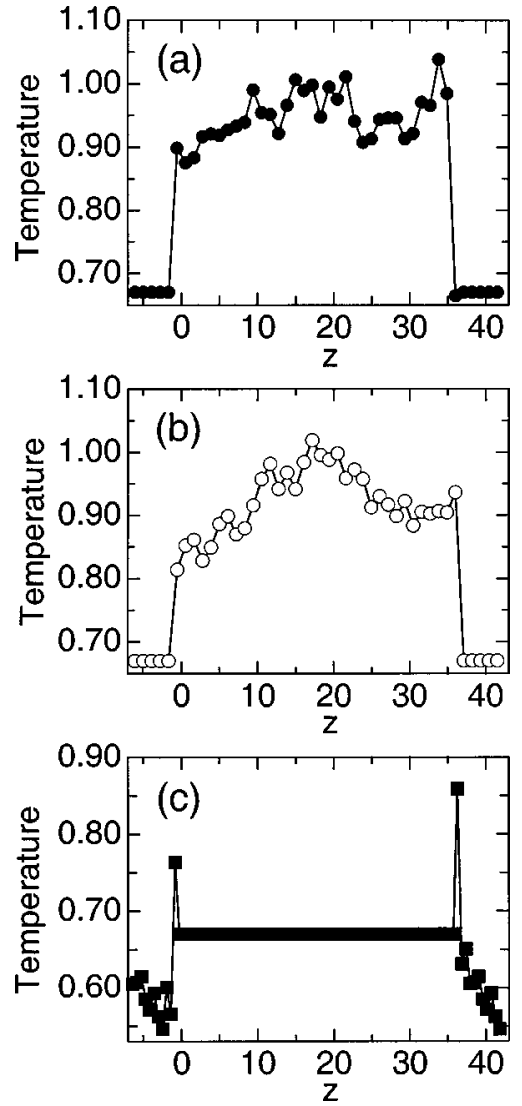

FIG. 4. The temperature profiles in the $z$ direction at a steady state for the (a) target, (b) carrier-gas, and (c) wall particles (the atomic-wall model).

age gas density [Fig. 3(a)]. The local temperature of the target particles [Fig. 4(a)] is also higher near the wall, indicating that the target particles are moving faster near the strongly adsorbing wall.

For the carrier gas, the number density distribution in the $z$ direction is fairly uniform [Fig. 3(b)]. The local temperature of carrier-gas particles [Fig. 4(b)], however, is higher in the middle region of the pore. As in real nucleation experiments, the primary role of the carrier gas is to remove the latent heat, especially in the middle region of the pore..$^{22,23}$ This heat, being pumped away from the system by both the carrier gas and the walls, is mainly the latent heat generated due to the formation of nucleus of target particle. The heat transfer via the carrier-gas particles takes place outside the nucleus of the target particle since the carrier-gas particles do not like to mix with the target particles in the nucleus.

In Figs. 3(c) and 4(c) the local density and temperature of the thermostatted atomic walls are shown. Near the wall surfaces, the local temperature of both weakly and strongly adsorbing walls is higher than the averaged temperature of the wall, because of the dynamical collisions between the wall and target particles at the wall surface. In the course of simulation, the solid walls are thermostatted to allow the heat dissipated through the walls, as that in real experiments. Thus, as shown in Fig. 4(c) the local temperature in the middle region of the walls is lower than the average.

In Figs. 5(a) and 5(b) the density and temperature profiles of the target particles at a steady state are presented for 

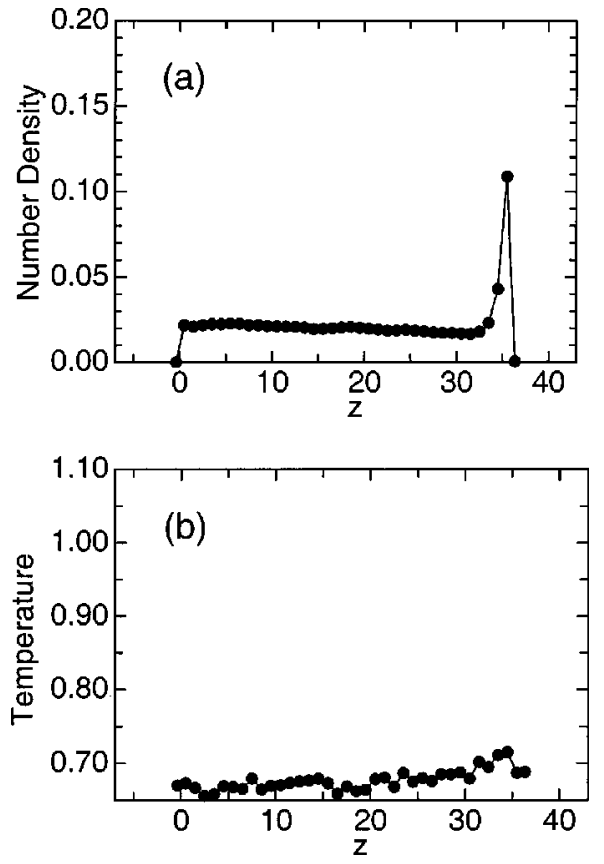

FIG. 5. (a) The density and (b) temperature profile at a steady state for the target particles (the smooth-wall model).

the smooth-wall model. In comparison with the same situation for the atomic-wall model [Figs. 3(a) and 4(a)], one can see that the overall density and temperature are lower.

\section{The nucleation rates}

The steady-state nucleation rate provides a measure of the dynamics of nucleus (or cluster) formation. In general, the nucleation rate is defined as the number of nuclei appearing per unit time and per volume. To evaluate the rate, we used the same method as in the case of homogeneous nucleation. ${ }^{15}$ It is worth noting that the cluster definition follows that of Stillinger's cluster, ${ }^{24}$ that is, a group of LJ particles can be considered as a cluster if every particle has at least one nearest neighbor within a distance of less than 1.5 (a value close to the first minimum of the pair correlation function of LJ liquid near the triple point).

In Figs. 6(a) and 6(b), the time development of the number of clusters larger than a critical size are shown for the atomic-wall and smooth-wall systems, respectively. It is found that the nucleation rate for the atomic-wall system is much faster than in the smooth-wall system. Moreover, the number of critical clusters in the atomic-wall system is larger than that in the smooth-wall system. The calculated rates of nucleation for both atomic-wall and smooth-wall systems are presented in Table I, where the nucleation rate at the steadystate $J$ is defined as the number of nuclei (larger than the critical nucleus) generated per unit volume per unit time. In the simulation the time evolution of the cluster-size distribution is recorded. Within a certain time window (typically, $10-100 \tau$, depending on the wall model), the system is in a quasisteady state. The rate is then determined by counting the number of clusters larger than the threshold. In Table I
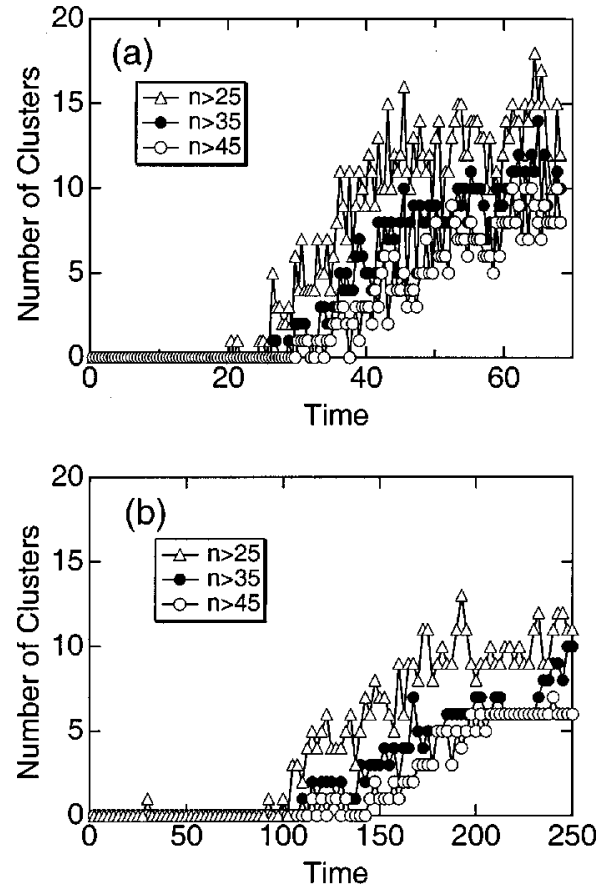

FIG. 6. The time development of the number of clusters larger than a critical size for the (a) atomic-wall and (b) smooth-wall system.

the estimated upper- $\left(J_{S}\right)$ and lower-bound $\left(J_{V}\right)$ values of the rate are presented for both the atomic-wall and smooth-wall systems.

Note that the nucleation rate for the atomic-wall system is about one order of magnitude higher than that for the smooth-wall system at the same condition, that is, the same temperature and vapor density. In other words, the use of a smooth-wall model underestimates the rate of heterogeneous nucleation. This indicates that the nucleation dynamics is quite sensitive to the wall model. A motion picture of the molecular dynamics can offer an insightful comparison of the nucleation process near the strongly adsorbing (atomic or smooth) wall. It is evident that the adsorption and desorption processes take place in a shorter period of time in the atomicwall system.

\section{Target-particle flux and diffusion}

One important mechanism of cluster formation in the heterogeneous nucleation ${ }^{10-14}$ is the surface diffusion of adsorbed particles on the strongly adsorbing surface. This was demonstrated in paper I using the smooth-wall system. Here, a similar analysis of target-particle flux and diffusion is undertaken. The results are then compared with those of smooth-wall system. We used the same notations of flux as in paper I.

TABLE I. The nucleation rates in the atomic-wall and smooth-wall system.

\begin{tabular}{ccc}
\hline \hline Wall model & $J_{S}$ & $J_{V}$ \\
\hline Atomic & $1.0 \times 10^{-4}$ & $2.8 \times 10^{-6}$ \\
Smooth & $1.5 \times 10^{-5}$ & $4.2 \times 10^{-7}$ \\
\hline \hline
\end{tabular}



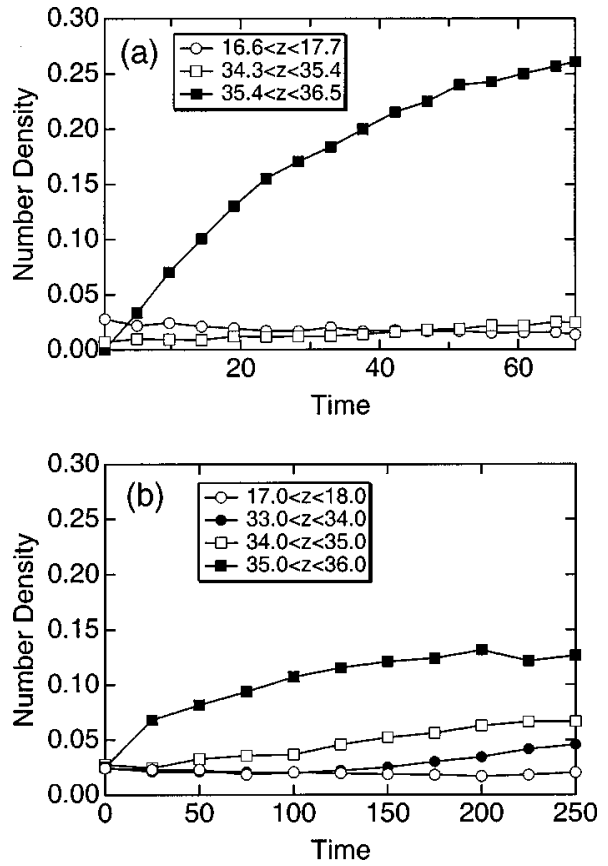

FIG. 7. The time development of the target-particle number density for the (a) atomic-wall and (b) smooth-wall system.

In Figs. 7(a) and 7(b) the time evolution of the local density and the monomer density (excluding all clusters) within two selected subdivisions of space between the walls are displayed for the atomic-wall and smooth-wall systems. One subdivision is the near-surface region $(34<z<36)$ and another is in the middle region of the pore $(16<z<18)$. Near the strongly adsorbing wall, regardless of the atomicwall or smooth-wall model, the local density of target particles always increases with time. However, in the atomicwall system the nucleation process is much faster. It is evident that the density-time development in the two selected subdivisions can be more easily distinguished for the atomic-wall system.

Next, we calculated the target-particle flux. As in paper I the target particles in the system are divided into four groups: group 1 includes all the monomers in the interior of the pore; group 2 includes all the monomers near the strongly adsorbing wall; group 3 includes all the clusters (excluding monomers) near the strongly adsorbing wall; group 4 include all the clusters (excluding monomers) in the interior of the pore. The net flux $J_{i j}$ can be defined as the rate of particle transformation from group $i$ to group $j(i, j=1$ -4 and $i \neq j$ ). In Figs. 8(a) and 8(b) the six flux curves are presented for the atomic-wall and smooth-wall system, respectively. The $J_{12}$ and $J_{13}$ describe adsorption kinetics of monomers toward the strongly adsorbing wall in the form of monomers and clusters, respectively. In the atomic-wall system, the dominant flux is $J_{12}$ and $J_{23}$ among the six. In particular, $J_{23}$ describes kinetics of the cluster formation on the wall via surface diffusion of adsorbed monomers. The $J_{43}$ describes the kinetics of cluster formation (near the strongly adsorbing wall) directly from the deposition of clusters already formed in the interior of the pore. This flux is the smallest one compared to the three fluxes mentioned above.
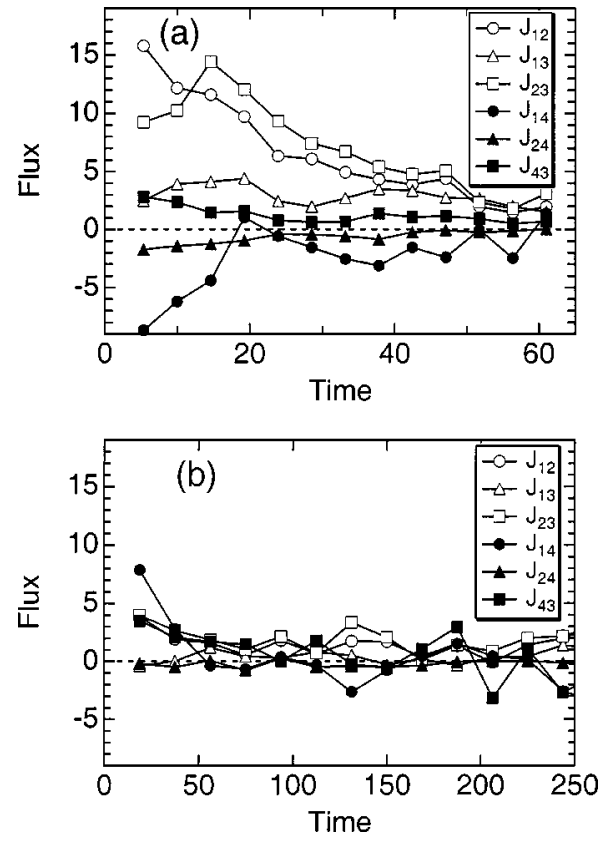

FIG. 8. The time development of various target-particle fluxes for the (a) atomic-wall and (b) smooth-wall system.

A possible explanation is that the clusters formed in the interior of the pore are mostly short lived. In contrast, in the smooth-wall system, the three fluxes, $J_{12}, J_{13}$ and $J_{23}$, are comparable in magnitude with each other, and with another flux as well.

Finally, we evaluated the diffusion coefficient of target particles in the two selected subdivisions inside the pore. The diffusion coefficient is obtained by calculating the meansquare displacement $\left\langle[r(t)-r(0)]^{2}\right\rangle$ of the target particles in the subdivision as a function of time. In Figs. 9(a) and
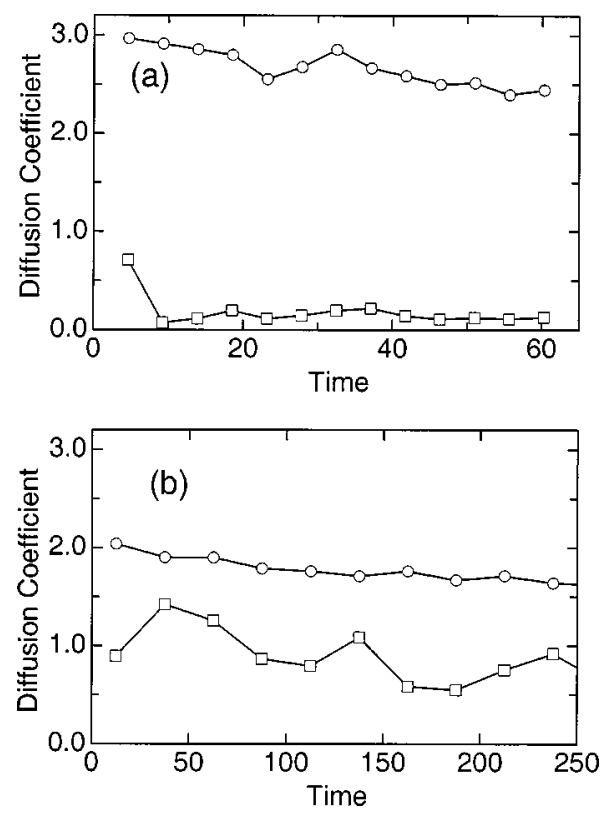

FIG. 9. The diffusion coefficient of the target particles for the (a) atomicwall and (b) smooth-wall system. The squares and circles represent the diffusion near the strongly adsorbing wall and in the interior of the pore, respectively. 
9(b) the diffusion coefficient is plotted as a function of time for the atomic-wall and smooth-wall system, respectively. The circles in Fig. 9 represent the particle diffusion in the interior of the pore while the squares represent the particle diffusion near the strongly adsorbing wall. In the atomic-wall system, it is found that target particles diffuse faster in the interior of the pore. This is consistent with the result that the local temperature of the target particles is higher in the atomic-wall system than that in the smooth-wall system [see Fig. 4(a) and Fig. 5(b)]. On the other hand, near the strongly adsorbing smooth wall, target particles diffuse faster. This is consistent with the result that the local density of the target particles is much lower in the smooth-wall system than that in the atomic-wall system [see Fig. 3(a) and Fig. 5(a)]. However, even with faster diffusion of target particles near the wall the nucleus formation is appreciably slower in the smooth-wall system, yielding a smaller local density near the strongly adsorbing wall (see Fig. 7).

\section{CONCLUSIONS}

We have carried out molecular dynamics simulation to investigate a heterogeneous nucleation process of supersaturated Lennard-Jones vapor in slit nanopores. In this study we extend paper I by treating the walls of slit as actual atomic walls serving as both the confining solid surfaces and a thermostat. As a result, the latent heat generated during nucleus formation can be dissipated through the walls, like in realworld experiments. Moreover, the atomic-wall model allows a more realistic simulation of the surface diffusion process of adsorbed particles toward nucleus formation.

A comparison with the previous study in paper I offers useful insight on the effects of the wall model to the nucleusformation dynamics. It is found that the adsorbed particles on the smooth wall diffuse faster than those on the atomic wall. However, the rate of nucleation is much faster in the atomic-wall system than in the smooth-wall system when both systems are at the same condition, that is, the same temperature and vapor density. In fact, the nucleation rate in the atomic-wall system can be one order of magnitude higher at the condition studied. In other words, the use of a smoothwall model can appreciably underestimate the rate of heterogeneous nucleation. Furthermore, motion pictures of molecular dynamics simulation provide a direct comparison of the nucleation process near the strongly adsorbing atomic and smooth walls. It is evident that the adsorption and desorption processes take place within a very short period of time in the atomic-wall system. Although in both wall-model systems the local density of target particles near the strongly adsorbing wall increases with time, the density-time development can be more easily distinguished in the atomic-wall system. All these results indicate that heterogeneous nucleation dynamics can be quite sensitive to the wall model.

We have also analyzed the target-particle flux and targetparticle diffusion in the slit. The results are compared with those of the smooth-wall system. It is found that the flux $J_{12}$ (flux of monomers from interior of the slit toward the strongly adsorbing wall) and $J_{23}$ (flux of cluster formed from surface diffusion of adsorbed monomers) in the atomic-wall system are much higher than those in the smooth-wall sys- tem. Moreover, among the six flux considered, $J_{12}$ and $J_{23}$ are much higher than others in the atomic-wall system whereas in the smooth-wall system $J_{12}$ and $J_{23}$ are comparable to others. Based on the flux analysis, we therefore conclude that the higher nucleation rate in the atomic-wall system appears due mainly to the greater magnitude of flux $J_{12}$ and $J_{23}$. It is also worth noting that in the atomic-wall system clusters formed in the interior of the slit are shorter lived. Consequently, the corresponding flux that describes the flux of clusters from the deposition of clusters already formed in the interior of the pore is the smallest one among all flux considered. The diffusion coefficient is calculated to examine effects of the wall model on the particle diffusion. In the atomic-wall system, the target particles diffuse faster in the interior of the pore. This is because the local temperature of the target particles is higher in that system. In the smooth-wall system, in contrast, target particles diffuse faster near the strongly adsorbing wall; yet the rate of nucleus formation is lower. Thus, we can conclude that the rate of nucleus formation on the wall is more sensitive to the kinetics of adsorption of gas particles than the diffusion rate of adsorbed particles on the wall, as far as for the wall systems considered here.

\section{ACKNOWLEDGMENTS}

We are grateful to Dr. K. J. Oh for helpful discussions. X.C.Z. thanks the National Science Foundation for support of this work.

${ }^{1}$ R. Evans, U. Marini Bettolo Marconi, and P. Tarazona, J. Chem. Phys. 84, 2376 (1986)

${ }^{2}$ H. K. Christenson, Phys. Rev. Lett. 73, 1821 (1994).

${ }^{3}$ K. Binder, D. P. Landau, and A. M. Ferrenberg, Phys. Rev. Lett. 74, 298 (1995).

${ }^{4}$ S. M. Gatica, M. M. Galbi, and M. W. Cole, Phys. Rev. E 59, 4484 (1999)

${ }^{5}$ F. Restagno, L. Bocquet, and T. Biben, Phys. Rev. Lett. 84, 2433 (2000).

${ }^{6}$ G. S. Heffelfinger, F. Van Swol, and K. E. Gubbins, Mol. Phys. 61, 1381 (1987).

${ }^{7}$ K. Yasuoka, G. T. Gao, and X. C. Zeng, J. Chem. Phys. 112, 4279 (2000).

${ }^{8}$ L. D. Gelb, K. E. Gubbins, R. Radhakrishnan, and M. SliwinskaBartkowiak, Rep. Prog. Phys. 62, 1573 (1999).

${ }^{9}$ D. W. Oxtoby, Acc. Chem. Res. 31, 91 (1998); V. Talanquer and D. W. Oxtoby, J. Chem. Phys. 114, 2519 (2001).

${ }^{10}$ K. C. Ho and J. R. Maa, J. Colloid Interface Sci. 85, 413 (1982).

${ }^{11}$ J. S. Sheu, J. R. Maa, and J. L. Katz, J. Stat. Phys. 52, 1143 (1988).

${ }^{12}$ J. S. Sheu and J. R. Maa, J. Colloid Interface Sci. 135, 178 (1990).

${ }^{13}$ H. Shinagawa, K. Okuyama, and Y. Kawamura, Can. J. Chem. Eng. 71, 238 (1993).

${ }^{14}$ M. Lazaridis, J. Colloid Interface Sci. 155, 386 (1993)

${ }^{15}$ K. Yasuoka and M. Matsumoto, J. Chem. Phys. 109, 8451 (1998).

${ }^{16}$ M. J. P. Nijmeijer, C. Bruin, A. F. Baker, and J. M. J. Van Leeuwen, Phys. Rev. A 42, 6052 (1990).

${ }^{17}$ S. Y. Liem, D. Brown, and J. H. R. Clarke, Phys. Rev. A 45, 3706 (1992).

${ }^{18}$ B. D. Todd, D. J. Evans, and P. J. Davis, Phys. Rev. E 52, 1627 (1995).

${ }^{19}$ S. T. Cui, P. T. Cummings, and H. D. Cochran, J. Chem. Phys. 111, 1273 (1999).

${ }^{20}$ W. Smith and T. R. Forester, Mol. Graphics 14, 136 (1993); The DL_POLY User's Manual. Version 2.11; Daresbury Laboratory, United Kingdom. http://www.dl.ac.uk/TCS/Software/DL_POLY, 1999.

${ }^{21}$ K. Kholmurodov, W. Smith, K. Yasuoka, and T. Ebisuzaki, Comput. Phys. Commun. 125, 167 (2000).

${ }^{22}$ A. Kantrowitz, J. Chem. Phys. 19, 1097 (1951).

${ }^{23}$ D. Kane, S. P. Fisenko, M. Rusyniak, and M. S. El-Shall, J. Chem. Phys. 111, 8496 (1999)

${ }^{24}$ F. H. Stillinger, J. Chem. Phys. 38, 1486 (1963). 